\title{
Spatiotemporal spread of Fermi-edge singularity as time-delayed interaction and impact on time-dependent RKKY-type coupling
}

\author{
Conor Jackson $\odot^{*}$ and Bernd Braunecker $\odot^{\dagger}$ \\ SUPA, School of Physics and Astronomy, University of St Andrews, North Haugh, St Andrews KY16 9SS, United Kingdom
}

(Received 9 June 2021; revised 24 January 2022; accepted 25 January 2022; published 14 February 2022)

\begin{abstract}
Fermi-edge singularity and Anderson's orthogonality catastrophe are paradigmatic examples of nonequilibrium many-body physics in conductors, appearing after a quench is created by the sudden change of a localized potential. We investigate if the signal carried by the quench can be used to transmit a long ranged interaction, reminiscent of the RKKY interaction, but with the inclusion of the full many-body propagation over space and time. We calculate the response of a conductor to two quenches induced by localized states at different times and locations. We show that building up and maintaining coherence between the localized states is possible only with finely tuned interaction between the localized states and the conductor. This puts bounds to the use of time controlled RKKY type interactions and may limit the speed at which some quantum gates could operate.
\end{abstract}

DOI: 10.1103/PhysRevResearch.4.013119

\section{INTRODUCTION}

The Fermi-edge singularity (FES) problem [1-4] and Anderson's orthogonality catastrophe (OC) [5] are the concepts behind one of the first and most important examples of how a quench can drive a strongly correlated quantum response of a fermionic conductor. For the OC the quench is caused by abruptly switching a localized scattering potential producing a proliferation of zero-energy particle-hole excitations. For the FES this is accompanied by the injection of an extra fermion into the conduction band, or its extraction. In both cases a screening cloud builds up near the potential that in the long time limit settles to a new ground state that is, up to the extra fermion for the FES, orthogonal to the initial ground state. The relaxation of the overlap of initial and final ground states follows a characteristic power law in time that depends only on the potential's scattering phase shift. Such a time dependence resembles the universal power-law responses of strongly correlated systems and makes FES/OC a model system for quantum critical behavior in the time domain. The Kondo effect in particular can be viewed as a superposition of OC cascades triggered by the Kondo spin flips [6]. Its universal many-body behavior has made FES an important testing ground for a multitude of many-body techniques over more than 50 years [1-19]. Experimental and theoretical evidence started with absorption and emission spectra in metals and semiconductors [20-28], and extended then to nanostructured systems [29-49] and atomic gases [50-55].

It is, however, notable that with few exceptions $[56,57]$ the focus has been on global response functions, and that there

\footnotetext{
${ }^{*}$ Corresponding author: cgj2@st-andrews.ac.uk

${ }^{\dagger}$ Corresponding author: bhb@st-andrews.ac.uk
}

Published by the American Physical Society under the terms of the Creative Commons Attribution 4.0 International license. Further distribution of this work must maintain attribution to the author(s) and the published article's title, journal citation, and DOI. is a major lack of investigation of the spatial build-up and spread of the FES. In this paper we show that the spatiotemporal spread offers a new perspective on FES physics, and we provide a systematic access. The FES quench can indeed be viewed as a coherent signal propagating through a fermionic bath. Picking up the signal at some distance causes a coupling with the source of the quench. We formalize this aspect and formulate the FES signal as a time delayed, long ranged effective interaction with a strong memory effect due to the slow power-law decay of response functions. The memory effect invalidates the use of an effective Hamiltonian so that we provide two appropriate formulations. One is fundamental in the form of a time dependent action on the Keldysh contour that incorporates the concept of time delayed interaction. The other one is formulated in the language of open system dynamics and concretely focuses on the time evolution of the density matrix. The focus on the density matrix is motivated by the requirements of quantum information processing. We illustrate the approaches through the example sketched in Fig. 1 where we investigate FES in combination with a quantum gate operation between two localized qubit type states. For concrete realizations one could consider a quantum wire coupled to two quantum dots or a fermionic atomic gas extending across two trapped charges. To transmit the signal we choose to inject a fermion into the conductor and extract it at a different location as this represents the simplest case of such a transmission that captures the relevant physics.

The time delay in the signal is due to the finite Fermi velocity $v_{F}$ and does not require the FES itself. The latter, however, causes a significant renormalization of the transmission amplitude and decoherence even after extraction unless special fine tuned conditions are met. This is in contrast the usual modeling of effective interactions carried through a different medium such as the RKKY interaction. These are permanently present and thus time and FES are of no significance. But if they are intended to be switched on and off as required for a quantum gate operation [58] our paper shows that time delay, entanglement with the conductor 


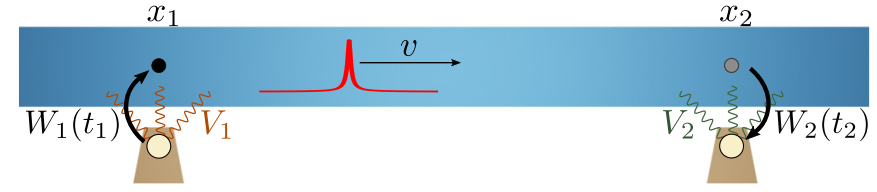

FIG. 1. Sketch illustrating the time nonlocal interaction mediated by a fermionic conductor, triggered by the tunneling transitions $W_{j}\left(t_{j}\right)$ at positions $x_{j}$ and times $t_{j}$ for $j=1,2$. The same transitions switch on the FES scattering potentials $V_{j}$ renormalizing the excitation peak traveling at velocity $v$.

and the FES are essential processes to be taken into account.

The structure of the remaining paper is as follows. In Sec. II we introduce the model represented in Fig. 1. In Sec. III we derive the time dependent action providing the conceptual picture for the time delayed interaction. Section IV contains the concrete calculation of the spatio-temporal response and its discussion. We conclude in Sec. V. Our results follow from calculations of a considerable length, as a side effect of such a mature research topic. To keep this work accessible we concentrate in the main text on presenting the physics of the spatio-temporal characteristics of FES with the discussion of its consequences, and we leave the proper calculations in the background. We thus use the appendices to provide the necessary methodologic and calculational details underpinning the discussion in a self-contained way. Appendix A contains the derivation of the path integral. In Appendix B we derive the shape of the evolution operator under the applied pulses, and in Appendix $\mathrm{C}$ we evaluate it explicitly. The structure of the amplitudes in the density matrix is derived in Appendix D, and in Appendix E we compute these amplitudes through the bosonization method.

\section{MODEL}

The minimal model shown in Fig. 1 consists of a Fermi gas with two localized states. For simplicity we consider spinless or spin polarized fermions, and we start with a noninteracting Hamiltonian

$$
H_{c}=\sum_{k} \epsilon_{k} c_{k}^{\dagger} c_{k}
$$

where $\epsilon_{k}$ is the dispersion and $c_{k}$ are the fermion operators, but an extension to interactions will be considered later. The localized states are single orbitals located at positions $x_{j}$ with operators $d_{j}$, energies $E_{j}$, and Hamiltonians

$$
H_{j}=E_{j} d_{j}^{\dagger} d_{j}
$$

for $j=1,2$. Transitions are induced by tunneling terms

$$
H_{W}=\sum_{j} W_{j}(t) d_{j}^{\dagger} \psi\left(x_{j}\right)+\text { H.c. }
$$

where $\psi(x)$ is the field operator corresponding to $c_{k}$, and $W_{j}(t)$ are time dependent amplitudes, applied over a range $\delta x_{W} \ll$ $\pi / k_{F}$ (with Fermi momentum $k_{F}$ ) such that tunneling can be expressed as point-like at $x_{j}$. The FES physics arises from the interaction

$$
H_{V}=V_{1} \psi^{\dagger}\left(x_{1}\right) \psi\left(x_{1}\right) d_{1} d_{1}^{\dagger}+V_{2} \psi^{\dagger}\left(x_{2}\right) \psi\left(x_{2}\right) d_{2}^{\dagger} d_{2} .
$$

Here we assume that initially level $d_{1}$ is occupied and $d_{2}$ empty such that tunneling out of $d_{1}$ and into $d_{2}$ switches on the scattering potentials $V_{j}$. Tunneling events are induced by sharp pump peaks $W_{j}(t)=W_{j} \delta\left(t-t_{j}\right)$ that trigger the FES and make the concept of a time delayed interaction between times $t_{1}$ and $t_{2}$ well defined. Such an operation comes also closest to controlling an interaction between the $d_{j}$ levels as required for a quantum gate operation. In the present case this could take the form of a conductor coupled to two quantum dot or defect states that are pulsed to induce injection and readout. We thus treat $H_{W}$ separately from the evolution under $H=H_{c}+H_{1}+H_{2}+H_{V}$

\section{TIME NONLOCAL INTERACTIONS}

To illustrate how FES appears in this interaction we consider the effective action between the $d_{j}$ levels obtained in the standard way from integrating out the conductor's degrees of freedom in a path integral formulation. To focus entirely on the physical interpretation we provide this derivation in Appendix A. For noninteracting fermions the action is quadratic in the $\psi(x)$ fields and the path integral is readily evaluated. This leads, in addition to the bare propagation under the $H_{j}$, to the effective action $S_{\text {eff }}=S_{L}+S_{C}$ with (setting $\hbar=1$ throughout)

$$
S_{L}=\sum_{j, j^{\prime}} \int_{\mathcal{K}} d t d t^{\prime} W_{j}(t) d_{j}^{\dagger}(t) G\left(x_{j}, t ; x_{j^{\prime}}, t^{\prime}\right) W_{j^{\prime}}\left(t^{\prime}\right) d_{j^{\prime}}\left(t^{\prime}\right),
$$

and

$$
S_{C}=-i \operatorname{Tr} \ln \left(G_{c} G^{-1}\right),
$$

which are derived in Eqs. (A8) and (A9). Here $G$ is the full fermion propagator in the presence of a path of the $d_{j}$ fields and $G_{c}$ the propagator for $d_{j} \equiv 0$. In the FES language of Ref. [4] $S_{L}$ is the open line propagator and $S_{C}$ is the closed loop sum expressing the $\mathrm{OC}$, which both are further resolved in space and include the two scattering centers $j=1,2$. The full time dependence is retained, and the time integrations run over the Keldysh contour $\mathcal{K}:-\infty \rightarrow+\infty \rightarrow-\infty$. The trace in $S_{C}$ involves integration over contour time and space.

The $W_{j}(t)$ control the paths $d_{j}(t)$ in $S_{\text {eff }}$ and thus the response to this interaction. Different paths of the $d_{j}(t)$ on the Keldysh branches $\mathcal{K}_{ \pm}: \mp \infty \rightarrow \pm \infty$ encode all possible FES scenarios. Notable first is the absence of OC for classical realizations of $d_{j}(t)$, which are equal on $\mathcal{K}_{ \pm}$, and thus forward and backward time integrations are identical, akin to the linked cluster theorem. Any interesting FES/OC effect is thus quantum with different $d_{j}(t)$ on $\mathcal{K}_{ \pm}$. The overlap integrals of FES spectra or of Loschmidt echos are extreme examples with $V_{j}(t)$ nonzero only on one branch. More general amplitudes involve the superposition of different paths, and a richer example of quantum interference, involving newly the spatio-temporal response too, is the off-diagonal density matrix element calculated below.

We observe that the time delayed interaction is carried only through the Green's function $G\left(x_{j}, t ; x_{j^{\prime}}, t^{\prime}\right)$ in $S_{L}$, the open line contribution, as a consequence of the tunnel type interaction. This would be replaced by two-fermion propagators 
for density-density interactions in $H_{W}$ instead, but does not involve the $\mathrm{OC}$, which always acts as a ground-state shake-up. In a fermionic liquid the $(x, t)$ dependence of Green's functions is dominated by the Fermi edge cutoff, which causes the characteristic power-law decay. The peak of these power laws travels with $v_{F}$ and thus causes largest impact at a distance $x$ only at time $x / v_{F}$. The interaction $V_{j}$ modifies the power-law exponents through the characteristic phase shifts $\delta_{j}$. Thus the interaction described by $S_{\text {eff }}$ combines three effects: The finite velocity of the interaction peak, which does not involve FES physics, the renormalization of the peak shape by FES, which can enhance or weaken the signal, and the general decay by the OC, which is always detrimental unless its long time behavior can be switched off by fine tuning. To substantiate these observations we consider now a concrete calculation.

\section{TIME EVOLUTION OF DENSITY MATRIX}

We illustrate the impact of spatio-temporal FES on the reduced density matrix $\rho_{d}$ of the two $d_{j}$ states as a quantity relevant for quantum information processing that is accessible e.g. through quantum state tomography. Due to $W_{j} \delta\left(t-t_{j}\right)$ all times $t, t^{\prime}$ in the path integral are pinned to $t_{j}$ on the Keldysh branches $\mathcal{K}_{ \pm}$. The number of possible paths $d_{j}(t)$ is then small and the path integral is evaluated directly. It is advantageous to delay tracing out the $\psi(x)$ and write $\rho_{d}(t)=$ $\operatorname{Tr}_{c}\left\{U(t) \rho(0) U^{\dagger}(t)\right\}$, where $\rho(0)$ is the full initial density matrix, $U(t)$ the evolution operator, and $\operatorname{Tr}_{c}$ the trace over the conductor's degrees of freedom. In this way we rewrite the conceptual path integral in the language of open system dynamics of a reduced density matrix $\rho_{d}$. For the pulsed transitions the number of paths is small and in this formulation we can evaluate the contribution from each path directly. The placement of $U$ and $U^{\dagger}$ corresponds to the evolution on the two Keldysh branches. For the pulsed transitions the evolution operator takes the form $[59,60]$

$$
U(t)=e^{-i H_{0} t} e^{-i \hat{W}_{2}} e^{-i \hat{W}_{1}}
$$

at $t>t_{2}>t_{1}$, where

$$
\hat{W}_{j}=e^{-i H_{0} t_{j}}\left[W_{j} d_{j}^{\dagger} \psi\left(x_{j}\right)+\text { H.c. }\right] e^{i H_{0} t_{j}} .
$$

The effect of the pulses is thus entirely contained in the unitary operators $e^{-i \hat{W}_{j}}$, and in Appendix B we provide a discussion of the derivation of Eq. (7). It is easy to show furthermore (see Appendix C) that

$$
e^{-i \hat{W}_{j}}=\mathbb{1}-i \alpha_{j} \hat{W}_{j}-\beta_{j} \hat{W}_{j}^{2},
$$

with $\alpha_{j}=\sin \left(w_{j}\right) / w_{j}, \beta_{j}=\left[1-\cos \left(w_{j}\right)\right] / w_{j}^{2}$ and $w_{j} \sim$ $W_{j} / \sqrt{\delta x_{W}}$. This means that the exact result takes the form of a second-order perturbative expansion but with renormalized coefficients $\alpha_{j}$ and $\beta_{j}$. This is a direct consequence of fermion statistics and has the advantage that the number of paths $d_{j}(t)$ created remains small such that the full time evolution can be evaluated exactly.

The two short pulses are chosen by analogy with the switching on and off of an exchange interaction between two qubits as the basis of a quantum gate generating entanglement between two qubits [58]. For the transmitted signal we obtain a similar gate operation between the $d_{j}$ levels but also decoherence due to the continuum of the fermionic fluctuations, both being strongly affected by the FES. To illustrate we assume that initially $\rho_{d}(0)=|1,0\rangle\langle 1,0|$, where $\left|n_{1}, n_{2}\right\rangle$ is the occupation number basis for $n_{j}=0,1$ the eigenvalues of $d_{j}^{\dagger} d_{j}$. Applying a pulse on $d_{1}$ at $t_{1}$ followed by a pulse on $d_{2}$ at $t_{2}$ the density matrix at time $t>t_{2}>t_{1}$ takes the form

$$
\rho_{d}(t)=\left(\begin{array}{cccc}
D_{0} & 0 & 0 & 0 \\
0 & A & C^{*} & 0 \\
0 & C & B & 0 \\
0 & 0 & 0 & D_{1}
\end{array}\right),
$$

spanned in the basis $\{|0,0\rangle,|1,0\rangle,|0,1\rangle,|1,1\rangle\}$. The zeros arise from terms without particle conservation along the full Keldysh contour.

Initially $A=1$ and all other amplitudes are zero. To put the result in context we compare it with the effect of a conventional exchange interaction $H_{J}=J\left(d_{1}^{\dagger} d_{2}+d_{2}^{\dagger} d_{1}\right)$. Since $H_{J}^{3}=J^{2} H_{J}$ the evolution operator becomes $U_{J}(t)=e^{-i H_{J} t}=$ $\mathbb{1}-i \sin (J t) H_{J}-[1-\cos (J t)] H_{J}^{2} / J$, similarly to the form of $U(t)$. The resulting density matrix takes the same form as Eq. (10) with $A=\cos (J t), B=\sin (J t), C=i \sin (2 J t) / 2$, and $D_{0}=D_{1}=0$. The density matrix remains a pure state but entangles the qubits. Therefore, such an interaction can be used for a quantum gate, with the time of interaction $t$ being the control parameter.

The present case for $\rho_{d}$ has several crucial differences due to the coupling to a fluctuating fermionic continuum. Primarily it makes the evolution of $\rho_{d}(t)$ nonunitary, by the creation of entanglement with the continuum that is lost for entangling the qubits. This naturally causes decoherence but also the time retardation features by the FES and the interaction that are our focus here. These are illustrated through the coefficient $C$, expressing the only route to build up entanglement between the two $d_{j}$ states. The other coefficients involve only classical paths $d_{j}(t)$ as they are on the diagonal of $\rho_{d}(t)$ and thus must have identical sequences of $\hat{W}_{j}$ in the evolution operators to the left and right of $\rho_{d}(0)$, which means identical sequences on both branches of the Keldysh contour. As noted above this cancels the OC response and as a consequence these coefficients show no or only a weak FES dependence. On the other hand, coefficient $C$ is off diagonal and requires different numbers of operators $\hat{W}_{j}$ on the two Keldysh branches. Namely we have

$$
\begin{aligned}
C= & -\alpha_{1} \alpha_{2}\langle 1,0| \operatorname{Tr}_{c}\left\{e^{-i H t}\left(\mathbb{1}-\beta_{2} \hat{W}_{2}^{2}\right)\left(\mathbb{1}-\beta_{1} \hat{W}_{1}^{2}\right)\right. \\
& \left.\times \rho(0) \hat{W}_{1} \hat{W}_{2} e^{i H t}\right\}|0,1\rangle .
\end{aligned}
$$

The detailed derivation of this expression as well as expressions for the other coefficients is provided in Appendix D. Equation (11) is exact but the contributions in terms of $\beta_{1,2}$ provide only qualitatively similar corrections to the leading term so that in the discussion we shall focus on the leading expression only, whereas in the figures we plot the full expression. Since the operators on both Keldysh branches, to the left and right of $\rho(0)$ in Eq. (11), are different both FES and OC persist. With the $\hat{W}_{1} \hat{W}_{2}$ operators on the right to the initial density matrix $C$ takes indeed a form similar to the Loschmidt echo, characterized by the interference of the perturbed system with the free evolution, with the difference that at time $t_{2}$ instead of switching off the interaction a tunneling event takes 
place and the system evolves further to time $t$ (notice also that $C=0$ for $t<t_{2}$ ).

Since three times $t_{1}, t_{2}, t$ and two positions $x_{1}, x_{2}$ are involved the number of correlation functions is large for each amplitude in $\rho(t)$ but an analytic evaluation is possible. To maximize the amplitude of the transmitted interaction we consider a one-dimensional (1D) conductor in which excitations can run only to the left or the right. This allows us in addition to use the bosonization technique, which provides the most straightforward technique to access the FES physics [7,61-63] and allows us to include interactions in the $1 \mathrm{D}$ conductor as well. In 1D the interactions are entirely characterized through a parameter $K$ such that in the noninteracting case $K=1$, for repulsive interactions $0<K<1$, and for attractive interactions $K>1$. Considering the zerotemperature limit the coefficients of $\rho$ are then composed of power laws expressing the propagation of the fermion from $\left(x_{1}, t_{1}\right)$ to $\left(x_{2}, t_{2}\right)$. Through the FES the power-law exponents depend on the phase shifts $\delta_{j}=2 V_{j} K / v$ induced by the scattering potentials $V_{j}$ [7]. Here $v=v_{F} / K$ is the interaction renormalized Fermi velocity [61-63]. The detailed evaluation is a standard calculation of some length but does not contribute further to the discussion. We thus provide the details in Appendix E and analyze here instead the physics resulting from the spatio-temporal spread. We should notice though that we do not consider backscattering on the $W_{j}$ potentials. Although this scattering is relevant in $1 \mathrm{D}$ it matters for weakly interacting systems mostly at time scales that can be tuned to be longer than the times considered here, and its inclusion would unnecessarily obscure the results. Further technical but also more quantitative arguments are given in Appendix E.

To leading order in the power laws $C$ is expressed as

$$
\begin{aligned}
C= & -\alpha_{1} \alpha_{2} W_{1} W_{2} e^{-i \Delta E_{1}\left(t_{1}-t\right)} e^{-i \Delta E_{2}\left(t_{2}-t\right)} \\
& \times\left(e^{-i k_{F}\left(x_{1}-x_{2}\right)} C_{-}+e^{+i k_{F}\left(x_{1}-x_{2}\right)} C_{+}\right) / 2 \pi a,
\end{aligned}
$$

where $a$ is the short distance cutoff, and where the coefficients $C_{v}$ with $v= \pm$ arise from the injection of a right or left moving fermion with momenta near the Fermi momentum $v k_{F}$. If we introduce $g_{x, t}=(a-i x+i v t) / a$ as the power-law basis we have [see Eq. (E17)]

$$
\begin{aligned}
C_{v}= & g_{0, t_{1}-t}^{\frac{\delta_{1}}{\pi K}-\frac{2 \delta_{1}^{2}}{\pi^{2} K}} g_{0, t_{2}-t}^{-\frac{\delta_{2}}{\pi \pi^{2}}-\frac{2 \delta_{2}^{2}}{\pi^{2} K}} g_{x_{1}-x_{2}, 0}^{-\frac{v \delta_{1}}{\pi}+\frac{\delta_{1} \delta_{2}}{\pi^{2} K}} g_{x_{2}-x_{1}, 0}^{\frac{\nu \delta_{1}}{\pi}+\frac{\delta_{1} \delta_{2}}{\pi^{2} K}} \\
& \times g_{x_{1}-x_{2}, t_{1}-t_{2}}^{-\frac{(1+K v)^{2}}{2 \pi K}+\frac{\left(\delta_{1}-\delta_{2}\right)(1+K v)}{2 \pi}+\frac{\delta_{1} \delta_{2}}{\pi^{2} K}} g_{x_{1}-x_{2}, t_{1}-t}^{\frac{\delta_{2}(1+K \nu)}{2 \pi K}-\frac{\delta_{1} \delta_{2}}{\pi^{2} K}} \\
& \times g_{x_{2}-x_{1}, t_{1}-t_{2}}^{-\frac{(1-K v)^{2}}{2 \pi K}+\frac{\left(\delta_{1}-\delta_{2}\right)(1-K v)}{2 \pi K}+\frac{\delta_{1} \delta_{2}}{\pi^{2} K}} g_{x_{2}-\delta_{2}(1-K v)}^{\frac{\delta_{1}, t_{1}-t}{\pi^{2} K}} \\
& \times g_{x_{1}-x_{2}, t_{2}-t}^{-\frac{\delta_{1}(1-K v)}{2 \pi K}}-\frac{\delta_{1} \delta_{2}}{\pi^{2} K} g_{x_{2}-x_{1}, t_{2}-t}^{-\frac{\delta_{1}(1+K v)}{2 \pi K}-\frac{\delta_{1} \delta_{2}}{\pi^{2} K}} .
\end{aligned}
$$

The multitude of power laws in Eq. (13) expresses the main result of this paper. It describes the various ways in which FES and OC shake up the conductor and propagate between the times $t_{1}, t_{2}$, and $t$, as well as between the positions $x_{1}$ and $x_{2}$, and how the interaction with $K \neq 1$ causes further fractionalization of the charge excitations. To understand the result let us consider first $t=t_{2}$, at which all dependence on $\delta_{2}$ vanishes as $V_{2}$ acts only for $t>t_{2}$. We

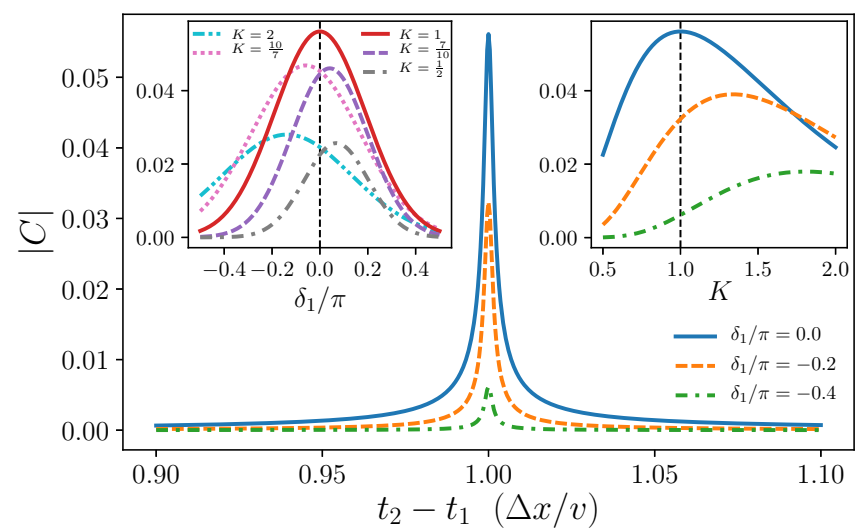

FIG. 2. Plot of coefficient $\left|C\left(t=t_{2}\right)\right|$ at $K=1$ for the indicated phase shifts $\delta_{1}$ for repulsive $V_{j}<0$ at $\Delta x=x_{2}-x_{1}=1000 a$ (using $a=v_{F}=1$ to set space and time units). The finite time-of-flight causes the sharp peak at $t_{2}-t_{1}=\Delta x / v$, and the FES its substantial suppression. The insets show the further suppression of the peak amplitude with interactions $K$ (right), and with $\delta_{1}$ for a selection of $K$ (left). Used parameters are $W_{j}=0.8 \sqrt{a}, n_{p}=0.4 / a, n_{h}=0.6 / a$, $\delta x_{W}=a$.

then have

$$
\begin{aligned}
C_{v}= & g_{0, t_{1}-t_{2}}^{\frac{\delta_{1}}{\pi K}-\frac{2 \delta_{1}^{2}}{\pi^{2} K}} g_{x_{1}-x_{2}, 0}^{-\frac{\delta_{1}(1+K \nu)}{2 \pi K}} g_{x_{2}-x_{1}, 0}^{-\frac{\delta_{1}(1-K \nu)}{2 \pi K}} \\
& \times g_{x_{1}-x_{2}, t_{1}-t_{2}}^{-\frac{(1+K \nu)^{2}}{4 K}+\frac{\delta_{1}(1+K \nu)}{2 \pi K}} g_{x_{2}-x_{1}, t_{1}-t_{2}}^{-\frac{(1-K \nu)^{2}}{4 K}+\frac{\delta_{1}(1-K \nu)}{2 \pi K}} .
\end{aligned}
$$

For $x_{2}-x_{1}=0$ this expression reduces further to the standard FES response whereas at nonzero $x_{2}-x_{1}$, as seen in Fig. 2, the last two factors produce a pronounced peak at $t_{2}-t_{1}=$ $\left|x_{2}-x_{1}\right| / v$ as consequence of the finite propagation velocity. Hence, in contrast to the instantaneous $H_{J}$ a fine tuning must be made to maximize the correlation between the two levels. Note that since the Hamiltonian is nonrelativistic tails of the correlators build up immediately and $C$ is nonzero already at all $t_{2}-t_{1}<\left|x_{2}-x_{1}\right| / v$.

Figure 2 also shows the substantial impact of FES. Varying $\delta_{1}$ from 0 to $\pm \pi / 2$ suppresses the peak amplitude by more than an order of magnitude, but in the tails we observe that the interplay between the OC and the contribution from the added fermion can lead to both larger or smaller amplitudes. The figure shows as well that interactions have a similar reducing effect but display also a partial compensation of the FES effect by shifting the maximum of $C$. Similar behavior can be found in the dynamics of a Kondo spin coupled to interacting chains [64] and is thus not specific for 1D.

Further distinction from a standard quantum gate arises entirely from the OC induced relaxation. This is the lasting effect of the correlation with the conductor and takes the role of an interaction that cannot just be switched off unless further fine tuning is achieved. Indeed $C \sim t^{-2\left(\delta_{1}+\delta_{2}\right)^{2} / \pi^{2} K}$ at long times $t \gg t_{2}$, suppressing with $C \rightarrow 0$ thus the transfer of information between the $d_{j}$ unless the $V_{j}$ are such that $\delta_{1}=-\delta_{2}$. The behavior as function of $t>t_{2}$ is shown for a selection of phase shifts and $K=1$ in Fig. 3 in which the condition $\delta_{1}=\delta_{2}=0$ leaves $C$ unchanged from its magnitude at $t=t_{2}$, as is indeed expected for a RKKY type coupling in a noninteracting system. For $\delta_{1}=-\delta_{2} \neq 0$ the amplitude 


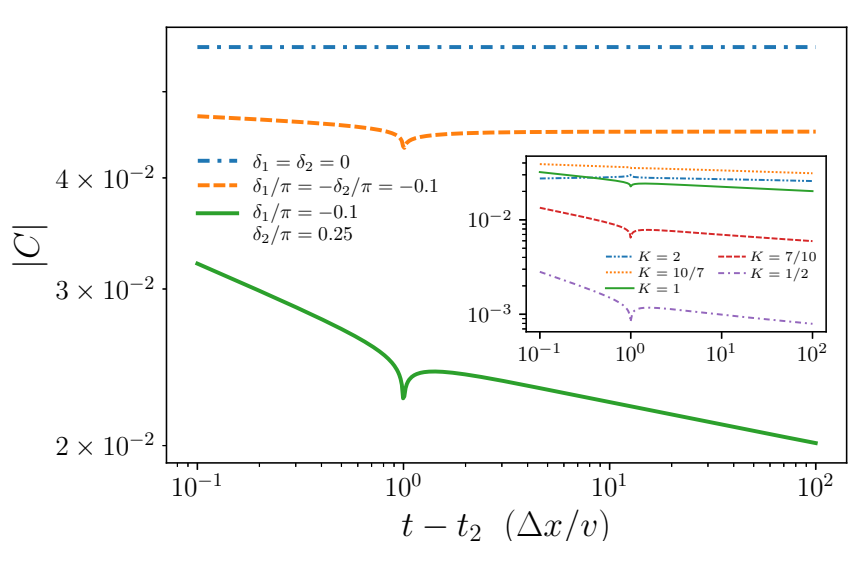

FIG. 3. Plot of $|C(t)|$ at $t>t_{2}$ for $K=1$ and parameters as in Fig. 2. For $\delta_{1}=-\delta_{2}$ coefficient $|C|$ becomes constant at large $t$ but decays for all other $\delta_{j}$. The inset shows the dependence on interactions $K$ for $\delta_{1} / \pi=-0.1$ and $\delta_{2} / \pi=0.25$.

saturates as noted at a constant value at large $t$. But the signal shows now a transient behavior with a satellite peak at $t-t_{2}=\left|x_{2}-x_{1}\right| / v$ caused by one of the last two factors in Eq. (13) and relies on $\delta_{1} \neq 0$. This effect arises due to the many-body interference of the OC at $x_{1}$ with the excitations created by the fermion absorption at $\left(x_{2}, t_{2}\right)$, manifesting as another Fermi-edge-style singularity. For $\delta_{1}+\delta_{2} \neq 0$ we see a similar transient behavior but then the further (albeit rather slow) decay with increasing $t$. Interactions with $K \neq 1$ are shown in the inset of Fig. 3. Here interactions can reduce and even enhance the amplitudes but do not change the qualitative features.

The time and space resolved spread of FES described by Eq. (13) therefore not just exhibits what could be thought as an obvious extension of the well known FES results to a peak traveling at finite velocity $v$. Instead all possible interference effects in time only, space only, and time and space mixed contribute each with a characteristic power-law exponent.

\section{CONCLUSIONS}

The results above provide an extension to our understanding of FES/OC physics to how the excitation extends nonlocally through space and time. We illustrated the impact on response functions through the example of correlated tunnel events, as a simple example of a quantum information type setup. With the finite propagation velocity the transition amplitudes are strongly peaked at the characteristic run time, and the FES causes a reduction of the peak amplitude together with the modification of the power-law tails. The OC generally remains detrimental to any long-term quantum coherence, even long after the second transition, unless the different scatterers are fine tuned to identical phase shifts. But even then there is a transient further decay before matrix elements of $\rho_{d}$ converge to a nonzero constant, which is the general result of the many interference processes in Eq. (13) distinguishing this case from the standard global FES/OC response. As the transient regime contains the most interesting coherent correlations, experimental probing would require ultrafast techniques, e.g., for electron conductors with $\mathrm{THz}$ resolu- tion. This could be offered by multidimensional spectroscopy [65-67], and could target the tomography of $\rho_{d}$ or the conductor's excitations directly. On the other hand if conductors are used for RKKY type interactions between quantum gates $[68,69]$, then sharp pulses triggering FES should be avoided and a smoother operation such as with minimal excitation pulses [70,71] should be chosen. This would necessarily slow down the rate at which such a gate could be operated. As a fundamental result, however, we have shown that the inclusion of a spatial component causes the FES response to split into all possible interference combinations between the involved time and space variables. While the OC diagrammatically remains decoupled from all other correlators and thus maintains its pure time dependence, all other FES processes connect the variables in a rather nontrivial manner, yet all with characteristic power laws.

\section{ACKNOWLEDGMENTS}

We thank H. Türeci for a stimulating discussion. C.J. acknowledges the support from the EPSRC under Grant No. $\mathrm{EP} / \mathrm{L} 015110 / 1$. The work presented in this paper is theoretical. No data were produced, and supporting research data are not required.

\section{APPENDIX A: PATH INTEGRAL FOR INTERACTION}

The derivation of the effective action for the time retarded interaction starts from the full action of the combined system of localized $d_{j}$ states and the continuum $\psi(x)$. If these $d_{j}$ and $\psi(x)$ represent now Grassmann fields the action reads $S=$ $S_{c}+\sum_{j=1,2} S_{j}+S_{V}+S_{W}$, with

$$
\begin{gathered}
S_{c}=\int_{\mathcal{K}} d t \int d x \psi^{\dagger}(x, t)\left(i \partial_{t}-H_{c}\right) \psi(x, t), \\
S_{j}=\int_{\mathcal{K}} d t d_{j}^{\dagger}(t)\left(i \partial_{t}-E_{j}\right) d_{j}(t), \\
S_{V}=-\int_{\mathcal{K}} d t V_{1} \psi^{\dagger}\left(x_{1}, t\right) \psi\left(x_{1}, t\right)\left[1-d_{1}^{\dagger}(t) d_{1}(t)\right] \\
-\int_{\mathcal{K}} d t V_{2} \psi^{\dagger}\left(x_{2}, t\right) \psi\left(x_{2}, t\right) d_{2}^{\dagger}(t) d_{2}(t), \\
S_{W}=\sum_{j=1,2} \int_{\mathcal{K}} d t W_{j}(t) \psi^{\dagger}\left(x_{j}, t\right) d_{j}(t)+\text { c.c. },
\end{gathered}
$$

in which the time integrals run over the Keldysh contour $\mathcal{K}:-\infty \rightarrow+\infty \rightarrow-\infty$. We consider only noninteracting fermions here in which $H_{c}$ is local and thus $S_{c}$ requires only a single spatial integral.

To obtain an effective action for the $d_{j}$ levels we integrate out the $\psi(x)$ fields as

$$
e^{i S_{\mathrm{eff}}\left[d_{j}^{\dagger}, d_{j}\right]}=\int D\left[\psi^{\dagger}, \psi\right] e^{i\left(S_{c}+S_{V}+S_{W}\right)},
$$

such that the total effective action is $\sum_{j} S_{j}+S_{\text {eff. }}$ In the absence of interactions, as considered for the evaluation of the path integral, the $\psi(x)$ integrals are Gaussians and the $\psi$ integration is straightforward,

$$
\int D\left[\psi^{\dagger}, \psi\right] e^{i\left(\psi \mid G^{-1} \psi\right)+(b \mid \psi)+(\psi \mid b)}=\operatorname{det}\left(i G^{-1}\right) e^{i(b \mid G b)},
$$


where the inner product $(\cdot \mid \cdot)$ consists of the $x$ and $t$ integrations. We write the Green's function in the kernel as $G^{-1}=$ $G_{c}^{-1}-V$, with $G_{c}^{-1}\left(x, t ; x^{\prime}, t^{\prime}\right)=\delta\left(t-t^{\prime}\right) \delta\left(x-x^{\prime}\right)\left[i \partial_{t}-H_{c}\right]$ and $\quad V\left(x, t ; x^{\prime}, t^{\prime}\right)=\delta\left(t-t^{\prime}\right)\left\{V_{1} \delta\left(x-x_{1}\right) \delta\left(x^{\prime}-x_{1}\right)[1-\right.$ $\left.\left.d_{1}^{\dagger}(t) d_{1}(t)\right]+V_{2} \delta\left(x-x_{2}\right) \delta\left(x^{\prime}-x_{2}\right) d_{2}^{\dagger}(t) d_{2}(t)\right\}$, and the inhomogeneous terms as $b(x, t)=\sum_{j} W_{j}(t) \delta\left(x-x_{j}\right) d_{j}(t)$.

The determinant factor can be written in the form

$$
\operatorname{det}\left(i G^{-1}\right)=\operatorname{det}\left(i G_{c}^{-1}\right) \exp \left(\operatorname{Tr} \ln \left(G_{c} G^{-1}\right)\right) .
$$

Here $\operatorname{det}\left(i G_{c}^{-1}\right)$ is an unimportant constant that can be dropped. On the other hand $e^{i S_{C}}=\exp \left(\operatorname{Tr} \ln \left(G_{c} G^{-1}\right)\right)$ is important. If we write it as

$$
S_{C}=-i \operatorname{Tr} \ln \left(1+i G_{c} V\right)
$$

we see from expanding the logarithm that it describes the full set of simple closed loop diagrams connecting vertices $V$. This term therefore incorporates the closed loop contribution responsible for the OC [4]. The full effective action then becomes $S_{\text {eff }}=S_{L}+S_{C}$ with

$$
S_{L}=\sum_{j, j^{\prime}} \int d t d t^{\prime} W_{j}(t) d_{j}^{\dagger}(t) G\left(x_{j}, t ; x_{j^{\prime}}, t^{\prime}\right) W_{j^{\prime}}\left(t^{\prime}\right) d_{j^{\prime}}\left(t^{\prime}\right),
$$

in which $G$ is the full Green's function on the Keldysh contour including the scattering on the time dependent potential $V$ created by the realizations of the $d_{j}(t)$ fields. In contrast to the closed loops in $S_{C}$ the propagator is pinned to the times $t, t^{\prime}$ at which the pulses $W_{j}$ are active and thus $S_{L}$ generalizes the open line diagrams of the FES [4].

\section{APPENDIX B: EVOLUTION OPERATOR FOR DELTA FUNCTION PULSES}

We consider a time dependent Hamiltonian of the form

$$
H_{\text {full }}(t)=H+W \delta\left(t-t_{1}\right),
$$

with $H=H_{c}+\sum_{j} H_{j}+H_{V}$ time independent and $W$ applied only through a pulse at time $t_{1}$ that is sharp enough to be treated as a delta function pulse. Such a time dependence allows for a simple solution for the evolution operator $U(t)$ which, however, requires some care. Indeed integrating the equation of motion $i \partial_{t} U(t)=H_{\text {full }}(t) U(t)$ over times $t_{1}-\delta t<t<t_{1}+\delta t$ for some $\delta t>0$ produces $U\left(t_{1}+\delta t\right)-$ $U\left(t_{1}-\delta t\right)=-i W U(t)$. The fact that the right hand side is nonzero shows on the left hand side that $U(t)$ is discontinuous at $t_{1}$. This in turn makes the right hand side ambiguous. The correct treatment of this situation is an old problem and, for instance, in Refs. [59,60] a thorough discussion is provided.

It turns out that the naive solution produces the correct answer. If we solve the equation of motion in the usual way by going to the interaction picture with $W$ as perturbation and perform a formal integration we obtain the standard form of the time ordered exponential

$$
U(t)=e^{-i H t} T \exp \left(-i \int_{0}^{t} d t^{\prime} \hat{W}\left(t^{\prime}\right) \delta\left(t^{\prime}-t_{1}\right)\right),
$$

where $T$ is the time ordering operator and $\hat{W}(t)=e^{-i H t} W e^{i H t}$. The implicit but far from obvious assumption in Eq. (B2) is that $T$ commutes with the integration. Accepting it though allows us immediately evaluate the integral in the exponential. Noting then that $T$ has no effect for equal time expressions we obtain

$$
U(t)=e^{-i H t} e^{-i \hat{W}\left(t_{1}\right)}=e^{-i H\left(t-t_{1}\right)} e^{-i W} e^{-i H t_{1}},
$$

for $t>t_{1}$ and $U(t)=e^{-i H t}$ for $t<t_{1}$. Although a rigorous treatment requires a more refined approach [59,60], Eq. (B3) is indeed the correct result. The last part of the equation provides the appropriate physical picture: The system evolves under $H$ before and after $t_{1}$, and the effect of the pulse is entirely contained in the unitary and nonperturbative operator $e^{-i W}$.

From the latter expression it is straightforward to obtain the evolution operator for sequences of pulses. Considering two pulses, $H_{\text {full }}=H+W_{1} \delta\left(t-t_{1}\right)+W_{2} \delta\left(t-t_{2}\right)$, we have

$$
\begin{aligned}
U(t) & =e^{-i H\left(t-t_{2}\right)} e^{-i W_{2}} e^{-i H\left(t_{2}-t_{1}\right)} e^{-i W_{1}} e^{-i H t_{1}} \\
& =e^{-i H t} e^{-i \hat{W}_{2}\left(t_{2}\right)} e^{-i \hat{W}_{1}\left(t_{1}\right)},
\end{aligned}
$$

where we have assumed $t>t_{2}>t_{1}$. Further pulses chain up in the same way.

\section{APPENDIX C: EVALUATION OF THE PULSE OPERATORS}

The operators $e^{-i \hat{W}_{j}}$ in the evolution operator resulting from pulses at times $t_{j}$ can be given a closed form in which we only need to be careful with infinities. The point-tunneling expressions

$$
\hat{W}_{j}=e^{i H t_{j}}\left(W_{j} d_{j}^{\dagger} \psi\left(x_{j}\right)+\text { H.c. }\right) e^{-i H t_{j}}
$$

cause at higher powers in the expansion of $e^{-i \hat{W}_{j}}$ products of $\psi\left(x_{j}\right)$ and $\psi^{\dagger}\left(x_{j}\right)$ that through the anticommutation rule $\left\{\psi(x), \psi^{\dagger}\left(x^{\prime}\right)\right\}=\delta\left(x-x^{\prime}\right)$ cause divergences. It thus must be noted that Eq. (C1) itself is only a convenient limit of the more general interaction

$$
\hat{W}_{j}=e^{i H t_{j}} \int d x\left(W_{j}(x) d_{j}^{\dagger} \psi(x)+\text { H.c. }\right) e^{-i H t_{j}},
$$

where $W_{j}(x)$ is a spatially dependent potential that is sharply peaked at $x=x_{j}$ and integrates to the amplitude $W_{j}$ in Eq. (C1). The order $\hat{W}_{j}^{2}$ itself would be unproblematic even with Eq. (C1). Using Eq. (C2) we find that

$$
\begin{aligned}
\hat{W}_{j}^{2}= & e^{i H t_{j}} \int d x d x^{\prime} W_{j}(x) W_{j}\left(x^{\prime}\right) \\
& \times\left(d_{j}^{\dagger} d_{j} \psi(x) \psi^{\dagger}\left(x^{\prime}\right)+d_{j} d_{j}^{\dagger} \psi^{\dagger}(x) \psi\left(x^{\prime}\right)\right) e^{-i H t_{j}} \\
\approx & W_{j}^{2} e^{i H t_{j}}\left(d_{j}^{\dagger} d_{j} \psi\left(x_{j}\right) \psi^{\dagger}\left(x_{j}\right)+d_{j} d_{j}^{\dagger} \psi^{\dagger}\left(x_{j}\right) \psi\left(x_{j}\right)\right) e^{-i H t_{j}},
\end{aligned}
$$

where the second line would also follow from Eq. (C1) and can be used for the practical evaluation of $\hat{W}_{j}^{2}$. For $\hat{W}_{j}^{3}$, however, we obtain

$$
\begin{aligned}
\hat{W}_{j}^{3}= & e^{i H t_{j}} \int d x d x^{\prime} d x^{\prime \prime} W_{j}(x) W_{j}\left(x^{\prime}\right) W_{j}\left(x^{\prime \prime}\right) \\
& \times\left(d_{j}^{\dagger} d_{j} d_{j}^{\dagger} \psi(x) \psi^{\dagger}\left(x^{\prime}\right) \psi\left(x^{\prime \prime}\right)+d_{j} d_{j}^{\dagger} d_{j} \psi^{\dagger}(x) \psi\left(x^{\prime}\right)\right. \\
& \left.\times \psi^{\dagger}\left(x^{\prime \prime}\right)\right) e^{-i H t_{j}} .
\end{aligned}
$$


Through the anticommutation relations for the $\psi(x)$ and $d_{j}$ this expression can then be reduced to

$$
\begin{aligned}
\hat{W}_{j}^{3}= & e^{i H t_{j}} \int d x d x^{\prime} W_{j}(x) W_{j}^{2}\left(x^{\prime}\right) \\
& \times\left(d_{j}^{\dagger} \psi(x)+d_{j} \psi^{\dagger}(x)\right) e^{-i H t_{j}}=w_{j}^{2} \hat{W}_{j},
\end{aligned}
$$

with

$$
w_{j}^{2}=\int d x W_{j}^{2}(x)
$$

Without the $x$ integrations this expression would have been left with the complication of diverging anticommutators that would have required an unnecessary cure, for instance, through point splitting. From the two results above it follows immediately that $\hat{W}_{j}^{2 n+1}=w_{j}^{2 n} \hat{W}_{j}$ and $\hat{W}_{j}^{2 n}=w_{j}^{2 n-2} \hat{W}_{j}^{2}$ for integer $n$. Consequently we have

$$
e^{-i \hat{W}_{j}}=\mathbb{1}-i \alpha_{j} \hat{W}_{j}-\beta_{j} \hat{W}_{j}^{2},
$$

with

$$
\begin{gathered}
\alpha_{j}=\sin \left(w_{j}\right) / w_{j}, \\
\beta_{j}=\left[1-\cos \left(w_{j}\right)\right] / w_{j}^{2} .
\end{gathered}
$$

This means that the exact form of $e^{-i \hat{W}_{j}}$ looks like its secondorder expansion with renormalized amplitudes. Notably, if we let $w_{j} \rightarrow 0$ then $\alpha_{j} \rightarrow 1$ and $\beta_{j} \rightarrow 1 / 2$, matching the secondorder amplitudes. Unitarity of Eq. (C7) imposes furthermore that

$$
\alpha_{j}^{2} \hat{W}_{j}^{2}=2 \beta_{j} \hat{W}_{j}^{2}-\beta_{j}^{2} \hat{W}_{j}^{4} .
$$

By the same methods that brought Eq. (C4) to Eq. (C5) we verify that indeed $\hat{W}_{j}^{4}=w_{j}^{2} \hat{W}_{j}^{2}$, and unitary follows from the trigonometric identity $2 \beta_{j}-w_{j}^{2} \beta_{j}^{2}=\alpha_{j}^{2}$.

\section{APPENDIX D: STRUCTURE OF DENSITY MATRIX AND CORRELATORS}

Let $\left|n_{1}, n_{2}\right\rangle$ denote the occupation state of the $d_{1}$ and $d_{2}$ levels. We assume that at time $t=0$ the localized states are in the $|1,0\rangle$ configuration and the fermionic conductor is in equilibrium. The initial density matrix is thus $\rho(0)=$ $|1,0\rangle\langle 1,0| \otimes \rho_{c}$, with $\rho_{c}$ the conductor's equilibrium density matrix. We apply the first $W_{1}$ pulse at time $t_{1}>0$ and the second $W_{2}$ pulse at time $t_{2}>t_{1}$. At any time $t>t_{2}$ the reduced density matrix takes the form

$$
\rho_{d}(t)=\left(\begin{array}{cccc}
D_{0} & 0 & 0 & 0 \\
0 & A & C^{*} & 0 \\
0 & C & B & 0 \\
0 & 0 & 0 & D_{1}
\end{array}\right),
$$

spanned in the basis $\{|0,0\rangle,|1,0\rangle,|0,1\rangle,|1,1\rangle\}$. The zeros arise from amplitudes that do not preserve the particle number in the fermionic conductor. (Note that here particle conservation must be considered only along the full Keldysh contour; in real time the number of particles in the conductor is allowed to vary.) The nonzero amplitudes at times $t>t_{2}>t_{1}$ are given by

$$
\begin{aligned}
& A=\langle 1,0| \operatorname{Tr}_{c}\left\{e^{-i H t}\left(\mathbb{1}-\beta_{2} \hat{W}_{2}^{2}\right)\left(\mathbb{1}-\beta_{1} \hat{W}_{1}^{2}\right) \rho(0)\right. \\
&\left.\times\left(\mathbb{1}-\beta_{1} \hat{W}_{1}^{2}\right)\left(\mathbb{1}-\beta_{2} \hat{W}_{2}^{2}\right) e^{i H t}\right\}|1,0\rangle, \\
& B=\alpha_{1}^{2} \alpha_{2}^{2}\left\langle 0,1\left|\operatorname{Tr}_{c}\left\{e^{-i H t} \hat{W}_{2} \hat{W}_{1} \rho(0) \hat{W}_{1} \hat{W}_{2} e^{i H t}\right\}\right| 0,1\right\rangle, \\
& C=- \alpha_{1} \alpha_{2}\langle 1,0| \operatorname{Tr}_{c}\left\{e^{-i H t}\left(\mathbb{1}-\beta_{2} \hat{W}_{2}^{2}\right)\left(\mathbb{1}-\beta_{1} \hat{W}_{1}^{2}\right)\right. \\
& \times\left.\rho(0) \hat{W}_{1} \hat{W}_{2} e^{i H t}\right\}|0,1\rangle, \\
& D_{0}= \alpha_{1}^{2}\langle 0,0| \operatorname{Tr}_{c}\left\{e^{-i H t}\left(\mathbb{1}-\beta_{2} \hat{W}_{2}^{2}\right) \hat{W}_{1} \rho(0) \hat{W}_{1}\right. \\
&\left.\times\left(\mathbb{1}-\beta_{2} \hat{W}_{2}^{2}\right) e^{i H t}\right\}|0,0\rangle, \\
& D_{1}= \alpha_{2}^{2}\langle 1,1| \operatorname{Tr}_{c}\left\{e^{-i H t} \hat{W}_{2}\left(\mathbb{1}-\beta_{1} \hat{W}_{1}^{2}\right) \rho(0)\right. \\
&\left.\times\left(\mathbb{1}-\beta_{1} \hat{W}_{1}^{2}\right) \hat{W}_{2} e^{i H t}\right\}|1,1\rangle .
\end{aligned}
$$

The evaluation of these amplitudes is done by keeping track of which tunneling transitions are nonzero on the $d_{j}$ levels, which gives rise to corresponding $\psi\left(x_{j}\right)$ or $\psi^{\dagger}\left(x_{j}\right)$ operators. The latter are then rearranged, using the cyclicity of $\operatorname{Tr}_{c}$ such that standard correlators $\langle\ldots\rangle=\operatorname{Tr}_{c}\left\{\ldots \rho_{c}\right\}$ with $\rho_{c}$, at the far right, are obtained. Expressions such as $W_{j}^{2}\left(\psi^{\dagger}\left(x_{j}\right) \psi\left(x_{j}\right)\right)^{2}$ are replaced by $w_{j}^{2} \psi^{\dagger}\left(x_{j}\right) \psi\left(x_{j}\right)$ as shown in Appendix C. Further simplifications are obtained through identity (C10) and the trigonometric relation between $\alpha_{j}$ and $\beta_{j}$.

In the expressions below we let $\psi_{j}=\psi\left(x_{j}\right)$ and use the notations $h_{0}=\left.H\right|_{V_{1}=V_{2}=0}, h_{1}=\left.H\right|_{V_{1} \neq 0, V_{2}=0}$, and $h_{12}=$ $\left.H\right|_{V_{1} \neq 0, V_{2} \neq 0}$. We then obtain

$$
\begin{gathered}
A=1-A_{1}-A_{2}+A_{3}+A_{4}, \\
A_{1}=\alpha_{1}^{2} W_{1}^{2}\left\langle e^{i h_{0} t_{1}} \psi_{1} \psi_{1}^{\dagger} e^{-i h_{0} t_{1}}\right\rangle \\
A_{2}=\alpha_{2}^{2} W_{2}^{2}\left\langle e^{i h_{0} t_{2}} \psi_{2}^{\dagger} \psi_{2} e^{-i h_{0} t_{2}}\right\rangle \\
A_{3}=4 \beta_{1} \beta_{2} W_{1}^{2} W_{2}^{2} \operatorname{Re}\left\langle e^{i h_{0} t_{2}} \psi_{2}^{\dagger} \psi_{2} e^{-i h_{0}\left(t_{2}-t_{1}\right)} \psi_{1} \psi_{1}^{\dagger} e^{-i h_{0} t_{1}}\right\rangle, \\
A_{4}=\beta_{1}^{2} \beta_{2}^{2} W_{1}^{4} w_{2}^{2} W_{2}^{2}\left\langle e^{i h_{0} t_{1}} \psi_{1} \psi_{1}^{\dagger} e^{-i h_{0}\left(t_{1}-t_{2}\right)} \psi_{2}^{\dagger} \psi_{2}\right. \\
\left.\times e^{-i h_{0}\left(t_{2}-t_{1}\right)} \psi_{1} \psi_{1}^{\dagger} e^{-i h_{0} t_{1}}\right\rangle
\end{gathered}
$$

which are all expressions independent of the scattering potentials $V_{j}$. The further diagonal entries are

$$
\begin{gathered}
B=\alpha_{1}^{2} \alpha_{2}^{2} W_{1}^{2} W_{2}^{2}\left\langle e^{i h_{0} t_{1}} \psi_{1} e^{-i h_{1}\left(t_{1}-t_{2}\right)} \psi_{2}^{\dagger} \psi_{2}\right. \\
\left.\times e^{-i h_{1}\left(t_{2}-t_{1}\right)} \psi_{1}^{\dagger} e^{-i h_{0} t_{1}}\right\rangle, \\
D_{0}=A_{1}-B, \\
D_{1}=A_{2}-A_{3}-A_{4} .
\end{gathered}
$$

While $D_{1}$ remains independent of the $V_{j}$ there is an explicit $V_{1}$ dependence in $B$ and $D_{0}$. However, all time dependence so far is pinned to the pulse times $t_{1}$ and $t_{2}$. The dependence on $t$ (for $t>t_{2}>t_{1}$ ) enters only through the off-diagonal component

$$
\begin{aligned}
C= & -\alpha_{1} \alpha_{2} W_{1} W_{2}\left\langle e^{i h_{0} t_{1}} \psi_{1} e^{-i h_{1}\left(t_{1}-t_{2}\right)} \psi_{2}^{\dagger}\right. \\
& \times e^{-i h_{12}\left(t_{2}-t\right)} e^{-i h_{0} t}
\end{aligned}
$$




$$
\begin{aligned}
& \times\left(1-\beta_{1} W_{1}^{2} e^{i h_{0} t_{1}} \psi_{1} \psi_{1}^{\dagger} e^{-i h_{0} t_{1}}\right) \\
& \left.\times\left(1-\beta_{2} W_{2}^{2} e^{i h_{0} t_{2}} \psi_{2}^{\dagger} \psi_{2} e^{-i h_{0} t_{2}}\right)\right\rangle,
\end{aligned}
$$

which is also the only term depending on $V_{2}$ as the latter potential is switched on only for times $t>t_{2}$.

The amplitudes $A_{1,2}$ can be evaluated immediately. Since $h_{0}$ does not perturb the ground state the correlator $\left\langle e^{i h_{0} t_{1}} \psi_{1} \psi_{1}^{\dagger} e^{-i h_{0} t_{1}}\right\rangle$ equals the local hole density $n_{h}$. Likewise $\left\langle e^{i h_{0} t_{2}} \psi_{2}^{\dagger} \psi_{2} e^{-i h_{0} t_{2}}\right\rangle$ gives the local particle density $n_{p}$. This leads to $A_{1}=\alpha_{1}^{2} W_{1}^{2} n_{h}$ and $A_{2}=\alpha_{2}^{2} W_{2}^{2} n_{p}$. The remaining amplitudes contain time propagating components and are evaluated through the bosonization technique.

\section{APPENDIX E: BOSONIZATION}

In the following we focus on one-dimensional (1D) systems such that the traveling signal remains directed and does not weaken its amplitude by expanding in a higher dimensional space. This has the additional advantage that we can use the bosonization technique [61-63], which a reliable method for the explicit evaluation of correlators. Bosonization allows us furthermore to quantitatively include the renormalization of system properties by interactions. We should emphasize, however, that we choose a 1D system and bosonization for convenience to provide explicit analytical results but we do not wish to lose universality by the restriction to the particular pure 1D physics. Indeed in many cases the interactions in $1 \mathrm{D}$ can cause a collective strong coupling response that qualitatively changes the system's properties. Such physics has been a central theme for 1D systems since many years but it is specific for this dimensionality. Notable is in particular that backscattering on the impurity causes an interactionindependent universal long time decay of the standard FES [10]. Yet here we explicitly exclude such strong coupling physics. We thus shall use bosonization in the same spirit as Schotte and Schotte [7] who mapped the radial expansion of a pure s-wave scattering in higher dimensions onto a 1D description solved by bosonization and thus could capture in such an elegant way the main many-body features of FES. For the present $1 \mathrm{D}$ description we shall keep nonetheless the fact that modes can travel to the right or the left and use a pure 1D description, but we either need to assume that backscattering on the impurity does not become relevant for the described physics (conditions are provided below), or that we deal with a quasi-1D system with sufficient degrees of freedom in the transverse directions such that the backscattering effect is reduced. Of course, purely $1 \mathrm{D}$ systems without backscattering can be realized experimentally as well such as through chiral quantum Hall edge states or helical edge states in topological insulators, and for such systems the description below can be applied with only straightforward adjustments.

The basic condition for bosonization is that the fermionic band is sufficiently filled such that one can consider the portions near the Fermi points $\pm k_{F}$ as two independent bands of right movers $R$ (near $k_{F}$ ) and left movers $L$ (near $\left.-k_{F}\right)$. The original fermion field operator is then written as $\psi(x)=e^{i k_{F} x} \psi_{R}(x)+e^{-i k_{F} x} \psi_{L}(x)$, where $\psi_{\nu}$ denotes the fermion operator on the $v=R, L$ movers branch. Furthermore the dispersion relation is linearized such that $\epsilon_{k, v} \approx v_{F}(k-$ $v k_{F}$ ), with the signs $v=R=+$ and $v=L=-$ replacing the letters $R, L$ where necessary. The resulting model is known as the Tomonaga-Luttinger model and is described by the Hamiltonian

$$
H_{T L}=\sum_{\nu} \int d x \psi_{\nu}^{\dagger}\left(-i v v_{F}\right) \partial_{x} \psi_{\nu}+H_{\mathrm{int}},
$$

in which we have chosen the chemical potential to be zero such that $H_{T L}$ measures the excitations about the ground state. The Hamiltonian $H_{\text {int }}$ contains the fermion-fermion interactions and can be expressed as

$$
H_{\mathrm{int}}=\sum_{\nu, v^{\prime}} \int d x d x^{\prime} \mathcal{V}\left(x-x^{\prime}\right) \psi_{\nu}^{\dagger}(x) \psi_{v^{\prime}}^{\dagger}\left(x^{\prime}\right) \psi_{\nu^{\prime}}\left(x^{\prime}\right) \psi_{v}(x),
$$

with $\mathcal{V}$ the interaction potential. In $H_{\text {int }}$ we have omitted terms that couple the $R$ and $L$ movers beyond the written density-density interaction. Indeed such terms are irrelevant in the renormalization group sense unless the fermion density is commensurate with the underlying lattice. We exclude such specific cases here, also in the spirit of the comments on the choice of a 1D model given above.

The mapping on bosonic degrees of freedom is then a standard procedure (see, e.g., Refs. [61-63] for an in depth discussion), with the boson fields representing density fluctuations of the $R$ and $L$ movers. The Hamiltonian (E1) becomes quadratic in the boson fields, and for a sufficiently short ranged (screened) interaction $\mathcal{V}$ such that the interaction is most pronounced within a range $<\pi / k_{F}$ all interactions can be treated as local. The Hamiltonian $H_{T L}$ then becomes a quadratic form described by a $2 \times 2$ matrix for the bosonic $R$ and $L$ fields with the off diagonal terms arising from the $R$ and $L$ density coupling in $H_{\text {int }}$. Such a matrix can be immediately diagonalized and the resulting eigenmodes, $\tilde{\phi}_{R, L}$, describe wave packets that still move only to the right or to the left, although when $\mathcal{V} \neq 0$ both contain contributions from both original $R$ and $L$ moving density waves. The Hamiltonian is written accordingly as $H_{T L}=\tilde{H}_{R}+\tilde{H}_{L}$ with

$$
\tilde{H}_{v}=\int d x \frac{v}{4 \pi K}\left(\partial_{x} \phi_{v}(x)\right)^{2},
$$

for $v=R, L$. Here $K$ encodes the interaction strength of $\mathcal{V}$, normalized such that $K=1$ corresponds to the noninteracting limit, $0<K<1$ to repulsive interactions and $K>1$ to attractive interactions, and $v$ is a renormalized Fermi velocity, often equal to $v=v_{F} / K$. The eigenmodes $\tilde{\phi}_{v}$ obey the commutation relations

$$
\left[\partial_{x} \tilde{\phi}_{v}\left(x^{\prime}\right), \tilde{\phi}_{v^{\prime}}(x)\right]=2 i \pi K v \delta_{v, v^{\prime}} \delta\left(x-x^{\prime}\right),
$$

such that $\tilde{\phi}_{v}$ and $\partial_{x} \tilde{\phi}_{v}$ are conjugate boson fields up to a normalization. In terms of the eigenmodes the original fermion operators are expressed as

$$
\psi_{\nu}(x)=\frac{\eta_{\nu}}{\sqrt{2 \pi a}} e^{-\frac{i}{2}\left(\nu-K^{-1}\right) \tilde{\phi}_{L}(x)-\frac{i}{2}\left(\nu+K^{-1}\right) \tilde{\phi}_{R}(x)},
$$

with $a$ a short distance cutoff, typically on the order of the lattice spacing. The $\eta_{v}$ are Klein factors, operators that lower the overall fermion number by one and guarantee fermionic 
exchange statistics. But for the further analysis they produce only unit expectation values and will be dropped.

The scattering potentials $V_{j}$ are in their fermionic form given by the Hamiltonian

$$
H_{V}=V_{1} \psi^{\dagger}\left(x_{1}\right) \psi\left(x_{1}\right) d_{1} d_{1}^{\dagger}+V_{2} \psi\left(x_{2}\right) \psi^{\dagger}\left(x_{2}\right) d_{2}^{\dagger} d_{2} .
$$

With the splitting into $R$ and $L$ movers $H_{V}$ has a forward scattering contribution remaining either in the $R$ or in the $L$ band, and a backscattering contribution transferring between $R$ and $L$. We shall neglect the latter, although this may seem counter-intuitive as backscattering produces a relevant FES response with a universal time decay that is independent of $V_{j}[10]$. Yet as mentioned above our main aim is to provide a description of the traveling FES signal and use bosonization as a convenient tool, but not to be limited to the particularities of the pure 1D response. In addition, even for the pure 1D case we should stress that the universal decay is a strong coupling response. It does not set in immediately but takes a characteristic time $\tau \sim\left(V_{j}^{b}\right)^{-1}\left(\xi / V_{j}^{b}\right)^{K /(1-K)}$ to build up before crossing over to the universal behavior [10]. Here $V_{j}^{b}=\left|V_{j}\left(2 k_{F}\right)\right|$ is the backscattering Fourier amplitude of $V_{j}$. The time $\tau$ is of significance mostly for strongly interacting systems with $K<0.7$ at which it can become very short. However, for typical scales as found in nanowires and not too strong interactions with $K>0.7$ the value of $\tau$ becomes on the order of microseconds or much larger such that the strong coupling limit from backscattering is not reached in the time scales governing the described physics otherwise. The physics then remains perturbative in the backscattering amplitude and has a direct $V_{j}$ dependence similar to the effect of forward scattering [10]. To capture the general effect of FES it is even in this pure 1D situation therefore sufficient to include only forward scattering.

In this case $H_{V}$ is expressed in terms of the boson fields as

$$
\begin{aligned}
H_{V}= & \sum_{\nu}\left[V_{1} \psi_{\nu}^{\dagger}\left(x_{1}\right) \psi_{v}\left(x_{1}\right) d_{1} d_{1}^{\dagger}+V_{2} \psi_{\nu}\left(x_{2}\right) \psi_{v}^{\dagger}\left(x_{2}\right) d_{2}^{\dagger} d_{2}\right] \\
= & \left\{\Delta E_{1}-\frac{V_{1}}{2 \pi K}\left[\partial_{x} \tilde{\phi}_{L}\left(x_{1}\right)+\partial_{x} \tilde{\phi}_{R}\left(x_{1}\right)\right]\right\} d_{1} d_{1}^{\dagger} \\
& +\left\{\Delta E_{2}-\frac{V_{2}}{2 \pi K}\left[\partial_{x} \tilde{\phi}_{L}\left(x_{2}\right)+\partial_{x} \tilde{\phi}_{R}\left(x_{2}\right)\right]\right\} d_{2}^{\dagger} d_{2},
\end{aligned}
$$

which incorporate the fluctuating parts of the forward scattering. Here $\Delta E_{j}=N V_{j}^{2} / 4 \pi v K$, with $N$ the system's particle number, are $d_{j}$ dependent energy shifts providing a ground state energy renormalization by the $V_{j}$ potentials. Since Eq. (E7) is linear in $\tilde{\phi}_{\nu}$ the total Hamiltonian $H_{T L}+H_{V}$ can be brought to the form of $H_{T L}$ by completing the square through a shift in the boson fields, $\partial_{x} \tilde{\phi}_{\nu}-\Delta$, such that the term proportional to $\left(\partial_{x} \tilde{\phi}_{v}\right) \Delta$ matches $H_{V}$. This can be performed on the operator level [7] by defining the shift operators

$$
\begin{aligned}
& \hat{T}_{1}=\exp \left(i \frac{\delta_{1}}{\pi K}\left[\tilde{\phi}_{R}\left(x_{1}\right)-\tilde{\phi}_{L}\left(x_{1}\right)\right] d_{1} d_{1}^{\dagger}\right), \\
& \hat{T}_{2}=\exp \left(i \frac{\delta_{2}}{\pi K}\left[\tilde{\phi}_{R}\left(x_{2}\right)-\tilde{\phi}_{L}\left(x_{2}\right)\right] d_{2}^{\dagger} d_{2}\right),
\end{aligned}
$$

where $\delta_{j}=2 K V_{j} / v$ is the scattering phase shift, which for the linearized spectrum matches the Born approximation [7]. Through the commutation relations (E4) we see that $H_{T L}+$
$H_{V}=T_{2}^{\dagger} T_{1}^{\dagger} H_{T L} T_{1} T_{2}+\Delta E_{1} d_{1} d_{1}^{\dagger}+\Delta E_{2} d_{2}^{\dagger} d_{2}$. If we let $T_{1}=$ $\left.\hat{T}_{1}\right|_{d_{1} d_{1}^{\dagger}=1}$ and $T_{2}=\left.\hat{T}_{2}\right|_{d_{2}^{\dagger} d_{2}=1}$ then it follows that $e^{-i h_{1} t}=$ $T_{1}^{\dagger} e^{-i h_{0} t} T_{1} e^{-i \Delta E_{1} t}$ and $e^{-i h_{12} t}=T_{2}^{\dagger} T_{1}^{\dagger} e^{-i h_{0} t} T_{1} T_{2} e^{-i\left(\Delta E_{1}+\Delta E_{2}\right) t}$, which allows us to write the correlators in $\rho_{d}$ entirely in terms of a time evolution under $h_{0}$. For instance, we have

$$
\begin{aligned}
C= & -\alpha_{1} \alpha_{2} W_{1} W_{2}\left\langle e^{i h_{0} t_{1}} \psi_{1} T_{1}^{\dagger} e^{-i\left(h_{0}+\Delta E_{1}\right)\left(t_{1}-t_{2}\right)} T_{1} \psi_{2}^{\dagger}\right. \\
& \times T_{2}^{\dagger} T_{1}^{\dagger} e^{-i\left(h_{0}+\Delta E_{1}+\Delta E_{2}\right)\left(t_{2}-t\right)} T_{1} T_{2} e^{-i h_{0} t} \\
& \times\left(1-\beta_{1} W_{1}^{2} e^{i h_{0} t_{1}} \psi_{1} \psi_{1}^{\dagger} e^{-i h_{0} t_{1}}\right) \\
& \left.\times\left(1-\beta_{2} W_{2}^{2} e^{i h_{0} t_{2}} \psi_{2}^{\dagger} \psi_{2} e^{-i h_{0} t_{2}}\right)\right\rangle .
\end{aligned}
$$

Inserting the time dependence $O(t)=e^{i h_{0} t} O e^{-i h_{0} t}$ for any operator $O$ the latter expression can be rewritten as

$$
\begin{aligned}
C= & -\alpha_{1} \alpha_{2} W_{1} W_{2} e^{-i \Delta E_{1}\left(t_{1}-t\right)} e^{-i \Delta E_{2}\left(t_{2}-t\right)} \\
& \times\left\langle\psi_{1}\left(t_{1}\right) T_{1}^{\dagger}\left(t_{1}\right) T_{1}\left(t_{2}\right) \psi_{2}^{\dagger}\left(t_{2}\right) T_{2}^{\dagger}\left(t_{2}\right) T_{1}^{\dagger}\left(t_{2}\right) T_{1}(t) T_{2}(t)\right. \\
& \times\left(1-\beta_{1} W_{1}^{2} \psi_{1}\left(t_{1}\right) \psi_{1}^{\dagger}\left(t_{1}\right)\right) \\
& \left.\times\left(1-\beta_{2} W_{2}^{2} \psi_{2}^{\dagger}\left(t_{2}\right) \psi_{2}\left(t_{2}\right)\right)\right\rangle .
\end{aligned}
$$

Similarly we find

$$
\begin{aligned}
B= & \alpha_{1}^{2} \alpha_{2}^{2} W_{1}^{2} W_{2}^{2}\left\langle\psi_{1}\left(t_{1}\right) T_{1}^{\dagger}\left(t_{1}\right) T_{1}\left(t_{2}\right) \psi_{2}^{\dagger}\left(t_{2}\right) \psi_{2}\left(t_{2}\right)\right. \\
& \left.\times T_{1}^{\dagger}\left(t_{2}\right) T_{1}\left(t_{1}\right) \psi_{1}^{\dagger}\left(t_{1}\right)\right\rangle,
\end{aligned}
$$

and corresponding expressions without $T_{j}$ operators for all other amplitudes in $\rho_{d}$. In all these expressions the fermion operators are replaced by Eq. (E5) and we note that $[61,62]$

$$
\begin{aligned}
\psi^{\dagger}(x) \psi(x)= & n_{p}-\frac{1}{2 \pi}\left[\partial_{x} \tilde{\phi}_{L}(x)+\partial_{x} \tilde{\phi}_{R}(x)\right] \\
& +\frac{1}{2 \pi a}\left(e^{i\left[\tilde{\phi}_{L}(x)+\tilde{\phi}_{R}(x)\right]}+\text { H.c. }\right),
\end{aligned}
$$

and $\psi(x) \psi^{\dagger}(x)=\left(n_{p}+n_{h}\right)-\psi^{\dagger}(x) \psi(x)$, where the densities $n_{p}$ and $n_{h}$ regularize the divergences from the delta function of the anticommutator.

The final evaluation of all correlators is done by using the identity $\left\langle\prod_{i} \exp \left(\lambda_{i} \tilde{\phi}_{i}\right)\right\rangle=\exp \left(\sum_{i<j} \lambda_{i} \lambda_{j} G_{i j}\right)$ valid for any theory with a quadratic bosonic Hamiltonian where $\tilde{\phi}_{i}=$ $\tilde{\phi}_{v_{i}}\left(x_{i}, t_{i}\right)$ and [63]

$$
\begin{aligned}
G_{i j} & =\left\langle\tilde{\phi}_{i} \tilde{\phi}_{j}-\left(\tilde{\phi}_{i}^{2}+\tilde{\phi}_{j}^{2}\right) / 2\right\rangle \\
& =-K \delta_{v_{i}, v_{j}} \ln \left[\frac{a-i v_{i}\left(x_{i}-x_{j}\right)+i v\left(t_{i}-t_{j}\right)}{a}\right] .
\end{aligned}
$$

In the latter equation we use the zero temperature $T=0$ limit, which is applicable as long as all considered time scales are shorter than the thermal time $\tau_{T}=2 \pi / k_{B} T$. For densitydensity correlators involving products of gradients we have

$$
\left\langle\partial_{x} \tilde{\phi}_{i} \partial_{x} \tilde{\phi}_{j}\right\rangle=\frac{K \delta_{v_{i}, v_{j}}}{\left[a-i v_{i}\left(x_{i}-x_{j}\right)+i v\left(t_{i}-t_{j}\right)\right]^{2}} .
$$

A subtlety arises from the term $\psi_{1}\left(t_{1}\right) \psi_{2}^{\dagger}\left(t_{2}\right) \psi_{2}\left(t_{2}\right) \psi_{1}^{\dagger}\left(t_{1}\right)$ in $B$, which must remain real and the standard point splitting method of bosonization is ambiguous. But there is no ambiguity in the noninteracting $K=1, \delta_{j}=0$ limit in which the evaluation of the fermionic amplitude is a basic application of Wick's theorem. From continuity with this result we find that 
in this case the correlators of the form of Eq. (E15) must instead be given by $-K \delta_{v_{i}, v_{j}} /\left\{a^{2}+\left[v_{i}\left(x_{i}-x_{j}\right)-v\left(t_{i}-t_{j}\right)\right]^{2}\right\}$.

The final results for the amplitudes $A, B, C, D_{0,1}$ are then obtained straightforwardly but require a good bookkeeping as they consist of products of many factors of the form $\left\{\left[a-i v_{i}\left(x_{i}-x_{j}\right)+i v\left(t_{i}-t_{j}\right)\right] / a\right\}^{\gamma_{i j}}$ with the various exponents $\gamma_{i j}$ arising from the $\psi$ and $T$ operators. We shall write out explicitly only the leading part of the amplitude $C$, without the higher order contributions proportional to $\beta_{1,2}$, as the discussion in the main text focuses on the latter. We have

$$
\begin{aligned}
C= & -\alpha_{1} \alpha_{2} W_{1} W_{2} e^{-i \Delta E_{1}\left(t_{1}-t\right)} e^{-i \Delta E_{2}\left(t_{2}-t\right)} \\
& \times\left(e^{-i k_{F}\left(x_{1}-x_{2}\right)} C_{-}+e^{+i k_{F}\left(x_{1}-x_{2}\right)} C_{+}\right) / 2 \pi a,
\end{aligned}
$$

in which the amplitudes $C_{v}$, for $v= \pm$ (denoting $R$ and $L$ movers, respectively), are products of the power laws arising from the multiple contractions between the boson fields. With $g_{x, t}=(a-i x+i v t) / a$ we have

$$
\begin{aligned}
& C_{\nu}=g_{0, t_{1}-t}^{\frac{\delta_{1}}{\pi K}-\frac{2 \delta_{1}^{2}}{\pi^{2} K}} g_{0, t_{2}-t}^{-\frac{\delta_{2}}{\pi K}-\frac{2 \delta_{2}^{2}}{\pi^{2} K}} g_{x_{1}-x_{2}, 0}^{-\frac{\nu \delta_{1}}{\pi}+\frac{\delta_{1} \delta_{2}}{\pi^{2} K}} g_{x_{2}-x_{1}, 0}^{\frac{\nu \delta_{1}}{\pi}+\frac{\delta_{1} \delta_{2}}{\pi^{2} K}} \\
& \times g_{x_{1}-x_{2}, t_{1}-t_{2}}^{-\frac{(1+K \nu)^{2}}{2 \pi K}+\frac{\left(\delta_{1}-\delta_{2}\right)(1+K v)}{2 \pi K}+\frac{\delta_{1} \delta_{2}}{\pi^{2} K}} g_{x_{1}-x_{2}, t_{1}-t}^{\frac{\delta_{2}(1+K v)}{2 \pi K}-\frac{\delta_{1} \delta_{2}}{\pi^{2} K}} \\
& \times g_{x_{2}-x_{1}, t_{1}-t_{2}}^{-\frac{(1-K \nu)^{2}}{2 \pi K}+\frac{\left(\delta_{1}-\delta_{2}\right)(1-K \nu)}{2 \pi K}+\frac{\delta_{1} \delta_{2}}{\pi^{2} K}} g_{x_{2}-x_{1}, t_{1}-t}^{\frac{\delta_{2}(1-K \nu)}{2 \pi K}-\frac{\delta_{1} \delta_{2}}{\pi^{2} K}} \\
& \times g_{x_{1}-x_{2}, t_{2}-t}^{-\frac{\delta_{1}(1-K \nu)}{2 \pi K}-\frac{\delta_{1} \delta_{2}}{\pi^{2} K}} g_{x_{2}-x_{1}, t_{2}-t}^{-\frac{\delta_{1}(1+K \nu)}{2 \pi K}-\frac{\delta_{1} \delta_{2}}{\pi^{2} K}} .
\end{aligned}
$$

The first two $x_{j}$ independent factors arise only from the OC. The next two time independent factors compensate in amplitude but provide a phase to the coefficient. The further terms encode the full spatio-temporal spread of the FES signal, with peaks whenever in $g_{x, t}$ the condition $x-v t=0$ is met. At $t=t_{2}$ this expression simplifies to

$$
\begin{aligned}
C_{v}= & g_{0, t_{1}-t_{2}}^{\frac{\delta_{1}}{\pi K}-\frac{2 \delta_{1}^{2}}{\pi^{2} K}} g_{x_{1}-x_{2}, 0}^{-\frac{\delta_{1}(1+K \nu)}{2 \pi K}} g_{x_{2}-x_{1}, 0}^{-\frac{\delta_{1}(1-K v)}{2 \pi K}} \\
& \times g_{x_{1}-x_{2}, t_{1}-t_{2}}^{-\frac{(1+K v)^{2}}{2 \pi K}} g_{x_{2}-x_{1}, t_{1}-t_{2}}^{-\frac{\delta_{1}(1+K v)}{4 \pi K}},
\end{aligned}
$$

in which all dependence on $\delta_{2}$ drops out.
[1] G. D. Mahan, Excitons in metals: Infinite hole mass, Phys. Rev. 163, 612 (1967).

[2] B. Roulet, J. Gavoret, and P. Nozières, Singularities in the $\mathrm{X}$-ray absorption and emission of metals. I. First-order parquet calculation, Phys. Rev. 178, 1072 (1969).

[3] P. Nozières, J. Gavoret, and B. Roulet, Singularities in the X-ray absorption and emission of metals. II. Self-consistent treatment of divergences, Phys. Rev. 178, 1084 (1969).

[4] P. Nozières and C. T. De Dominicis, Singularities in the x-ray absorption and emission of metals. III. One-body theory exact solution, Phys. Rev. 178, 1097 (1969).

[5] P. W. Anderson, Ground state of a magnetic impurity in a metal, Phys. Rev. 164, 352 (1967).

[6] G. Yuval and P. W. Anderson, Exact results for the Kondo problem: One-body theory and extension to finite temperature, Phys. Rev. B 1, 1522 (1970).

[7] K. D. Schotte and U. Schotte, Threshold behavior of the X-ray spectra of light metals, Phys. Rev. 185, 509 (1969).

[8] M. Combescot and P. Nozières, Infrared catastrophy and excitons in the X-ray spectra of metals, J. Phys. (Paris) 32, 913 (1971).

[9] K. Ohtaka and Y. Tanabe, Theory of the soft-x-ray edge problem in simple metals: Historical survey and recent developments, Rev. Mod. Phys. 62, 929 (1990).

[10] A. O. Gogolin, Local Time-Dependent Perturbation in Luttinger Liquid, Phys. Rev. Lett. 71, 2995 (1993).

[11] A. Komnik, R. Egger, and A. O. Gogolin, Exact Fermi-edge singularity exponent in a Luttinger liquid, Phys. Rev. B 56, 1153 (1997).

[12] B. Muzykantskii, N. d'Ambrumenil, and B. Braunecker, FermiEdge Singularity in a Nonequilibrium System, Phys. Rev. Lett. 91, 266602 (2003).

[13] D. A. Abanin and L. S. Levitov, Tunable Fermi-Edge Resonance in an Open Quantum Dot, Phys. Rev. Lett. 93, 126802 (2004).
[14] N. d'Ambrumenil and B. Muzykantskii, Fermi gas response to time-dependent perturbations, Phys. Rev. B 71, 045326 (2005).

[15] B. Braunecker, Response of a Fermi gas to time-dependent perturbations: Riemann-Hilbert approach at nonzero temperatures, Phys. Rev. B 73, 075122 (2006).

[16] E. Bettelheim, A. G. Abanov, and P. Wiegmann, Orthogonality Catastrophe and Shock Waves in a Nonequilibrium Fermi Gas, Phys. Rev. Lett. 97, 246402 (2006).

[17] H. E. Türeci, M. Hanl, M. Claassen, A. Weichselbaum, T. Hecht, B. Braunecker, A. Govorov, L. Glazman, A. Imamoglu, and J. von Delft, Many-Body Dynamics of Exciton Creation in a Quantum Dot by Optical Absorption: A Quantum Quench Towards Kondo Correlations, Phys. Rev. Lett. 106, 107402 (2011).

[18] I. Snyman, Electron-electron correlations in a dynamical impurity system with a Fermi edge singularity, Phys. Rev. B 87, 165135 (2013).

[19] I. Snyman and S. Florens, Microscopic bosonization of band structures: X-ray processes beyond the Fermi edge, New J. Phys. 19, 113031 (2017).

[20] R. Haensel, G. Keitel, P. Schreiber, B. Sonntag, and C. Kunz, Measurement of the Anomaly at the $L_{I I, I I I}$ Edge of Sodium, Phys. Rev. Lett. 23, 528 (1969).

[21] R. A. Tilton, D. J. Phelps, and C. P. Flynn, Optical Absorption Threshold in Dilute Alkali-Metal-Rare-Gas Alloys, Phys. Rev. Lett. 32, 1006 (1974).

[22] T. A. Callcott and E. T. Arakawa, Temperature Dependence of the K X-ray Emission Edge of Li, Phys. Rev. Lett. 38, 442 (1977).

[23] T. Ishii, Y. Sakisaka, S. Yamaguchi, T. Hanyu, and H. Ishii, Threshold singularities of p-shell absorption in potassium, rubidium and cesium metals, J. Phys. Soc. Jpn. 42, 876 (1977).

[24] C. P. Flynn, The optical excitation spectra of impurity atoms in metals and on metal surfaces, Surf. Sci. 158, 84 (1985). 
[25] G. K. Wertheim, D. M. Riffe, and P. H. Citrin, Bulk and surface singularity indices in the alkali metals, Phys. Rev. B 45, 8703 (1992).

[26] S. Jiang, J. M. Zhang, L. J. Zhang, and S. C. Shen, The Fermiedge singularity in CdTe/Cd1-xMnxTe:In multiple quantum wells, J. Phys.: Condens. Matter 6, 10391 (1994).

[27] V. M. Adamjan, J. Ortner, A. G. Salistra, and I. M. Tkachenko, $\mathrm{X}$-ray-absorption problem in metals within the one-electron approximation, Phys. Rev. B 52, 13827 (1995).

[28] C. A. Swarts and J. D. Dow, X-ray line shapes of metals: Exact solutions of a final-state interaction model, Phys. Rev. B 72, 155119 (2005).

[29] S. Schmitt-Rink, C. Ell, and H. Haug, Many-body effects in the absorption, gain, and luminescence spectra of semiconductor quantum-well structures, Phys. Rev. B 33, 1183 (1986).

[30] J. M. Calleja, A. R. Goñi, B. S. Dennis, J. S. Weiner, A. Pinczuk, S. Schmitt-Rink, L. N. Pfeiffer, K. W. West, J. F. Müller, and A. E. Ruckenstein, Large optical singularities of the one-dimensional electron gas in semiconductor quantum wires, Solid State Commun. 79, 911 (1991).

[31] K. A. Matveev and A. I. Larkin, Interaction-induced threshold singularities in tunneling via localized levels, Phys. Rev. B 46, 15337 (1992).

[32] A. K. Geim, P. C. Main, N. La Scala, L. Eaves, T. J. Foster, P. H. Beton, J. W. Sakai, F. W. Sheard, M. Henini, G. Hill, and M. A. Pate, Fermi-Edge Singularity in Resonant Tunneling, Phys. Rev. Lett. 72, 2061 (1994).

[33] D. H. Cobden and B. A. Muzykantskii, Finite-Temperature Fermi-Edge Singularity in Tunneling Studied Using Random Telegraph Signals, Phys. Rev. Lett. 75, 4274 (1995).

[34] I. E. Itskevich, T. Ihn, A. Thornton, M. Henini, T. J. Foster, P. Moriarty, A. Nogaret, P. H. Beton, L. Eaves, and P. C. Main, Resonant magnetotunneling through individual self-assembled InAs quantum dots, Phys. Rev. B 54, 16401 (1996).

[35] K. A. Benedict, A. S. Thornton, T. Ihn, P. C. Main, L. Eaves, and M. Henini, Fermi edge singularities in high magnetic fields, Phys. B: Condens. Matter 256-258, 519 (1998).

[36] I. Hapke-Wurst, U. Zeitler, H. Frahm, A. G. M. Jansen, R. J. Haug, and K. Pierz, Magnetic-field-induced singularities in spin-dependent tunneling through InAs quantum dots, Phys. Rev. B 62, 12621 (2000).

[37] M. Hentschel, D. Ullmo, and H. U. Baranger, Fermi edge singularities in the mesoscopic regime: Anderson orthogonality catastrophe, Phys. Rev. B 72, 035310 (2005).

[38] F. Liu, M. Bao, K. L. Wang, D. Zhang, and C. Zhou, Coulomb attractive random telegraph signal in a single-walled carbon nanotube, Phys. Rev. B 74, 035438 (2006).

[39] X. L. Wang, M. Ogura, T. Guillet, V. Voliotis, and R. Grousson, Strong Fermi-edge singularity in ultra-high-quality AlGaAs/GaAs quantum wires, Physica E 32, 329 (2006).

[40] M. Rüth, T. Slobodskyy, C. Gould, G. Schmidt, and L. W. Molenkamp, Fermi edge singularity in II-VI semiconductor resonant tunneling structures, Appl. Phys. Lett. 93, 182104 (2008).

[41] C. Latta, F. Haupt, M. Hanl, A. Weichselbaum, M. Claassen, W. Wuester, P. Fallahi, S. Faelt, L. Glazman, J. Von Delft, H. E. Türeci, and A. Imamoglu, Quantum quench of Kondo correlations in optical absorption, Nature (London) 474, 627 (2011).
[42] M. Heyl and S. Kehrein, Crooks Relation in Optical Spectra: Universality in Work Distributions for Weak Local Quenches, Phys. Rev. Lett. 108, 190601 (2012).

[43] N. Ubbelohde, K. Roszak, F. Hohls, N. Maire, R. J. Haug, and T. Novotný, Strong quantum memory at resonant Fermi edges revealed by shot noise, Sci. Rep. 2, 374 (2012).

[44] I. Chernii, I. P. Levkivskyi, and E. V. Sukhorukov, Fermi-edge singularity in chiral one-dimensional systems far from equilibrium, Phys. Rev. B 90, 245123 (2014).

[45] T. Krähenmann, L. Ciorciaro, C. Reichl, W. Wegscheider, L. Glazman, T. Ihn, and K. Ensslin, Fermi edge singularities in transport through lateral GaAs quantum dots, New J. Phys. 19, 23009 (2017).

[46] A. S. Goremykina and E. V. Sukhorukov, Fermi-edge singularity and related interaction induced phenomena in multilevel quantum dots, Phys. Rev. B 95, 155419 (2017).

[47] V. V. Ponomarenko and I. A. Larkin, Probing qubit dynamics at the tunneling Fermi-edge singularity with alternating current, Phys. Rev. B 95, 205416 (2017).

[48] J. K. Kühne and R. J. Haug, Scaling of the Fermi-Edge Singularity in Quantum Dots, Phys. Status Solidi 256, 1800510 (2019).

[49] V. V. Ponomarenko and I. A. Larkin, Charge transfer statistics and qubit dynamics at the tunneling Fermi-edge singularity, Phys. Rev. B 100, 085433 (2019).

[50] M. Knap, A. Shashi, Y. Nishida, A. Imambekov, D. A. Abanin, and E. Demler, Time-Dependent Impurity in Ultracold Fermions: Orthogonality Catastrophe and Beyond, Phys. Rev. X 2, 041020 (2012).

[51] A. Sindona, J. Goold, N. Lo Gullo, S. Lorenzo, and F. Plastina, Orthogonality Catastrophe and Decoherence in a TrappedFermion Environment, Phys. Rev. Lett. 111, 165303 (2013).

[52] B. Dóra, F. Pollmann, J. Fortágh, and G. Zaránd, Loschmidt Echo and the Many-Body Orthogonality Catastrophe in a Qubit-Coupled Luttinger Liquid, Phys. Rev. Lett. 111, 046402 (2013).

[53] S. Campbell, M. Á. García-March, T. Fogarty, and T. Busch, Quenching small quantum gases: Genesis of the orthogonality catastrophe, Phys. Rev. A 90, 013617 (2014).

[54] M. Schiró and A. Mitra, Transient Orthogonality Catastrophe in a Time-Dependent Nonequilibrium Environment, Phys. Rev. Lett. 112, 246401 (2014).

[55] W. E. Liu, J. Levinsen, and M. M. Parish, Variational Approach for Impurity Dynamics at Finite Temperature, Phys. Rev. Lett. 122, 205301 (2019).

[56] A. Sheikhan and I. Snyman, Fermi edge singularity and finitefrequency spectral features in a semi-infinite one-dimensional wire, Phys. Rev. B 86, 085122 (2012).

[57] I. Snyman, Linear response of a one-dimensional conductor coupled to a dynamical impurity with a Fermi edge singularity, Phys. Rev. B 89, 085118 (2014).

[58] D. Loss and D. P. DiVincenzo, Quantum computation with quantum dots, Phys. Rev. A 57, 120 (1998).

[59] M. G. Calkin, D. Kiang, and Y. Nogami, Proper treatment of the delta function potential in the one-dimensional Dirac equation, Am. J. Phys. 55, 737 (1987).

[60] M. Nedeljkov and M. Oberguggenberger, Ordinary differential equations with delta function terms, Publ. Inst. Math. 91, 125 (2012). 
[61] A. O. Gogolin, A. A. Nersesyan, and A. M. Tsvelik, Bosonization and Strongly Correlated Systems (Cambridge University Press, Cambridge, 1998).

[62] T. Giamarchi, Quantum Physics in One Dimension (Oxford University Press, Oxford, 2007).

[63] R. Shankar, Quantum Field Theory and Condensed Matter: An Introduction (Cambridge University Press, Cambridge, 2017).

[64] H. Bragança, M. F. Cavalcante, R. G. Pereira, and M. C. O. Aguiar, Quench dynamics and relaxation of a spin coupled to interacting leads, Phys. Rev. B 103, 125152 (2021).

[65] W. Kuehn, K. Reimann, M. Woerner, T. Elsaesser, and R. Hey, Two-dimensional terahertz correlation spectra of electronic excitations in semiconductor quantum wells, J. Phys. Chem. B 115, 5448 (2010).

[66] M. Maiuri, M. Garavelli, and G. Cerullo, Ultrafast spectroscopy: State of the art and open challenges, J. Am. Chem. Soc. 142, 3 (2020).
[67] J. Lloyd-Hughes, P. M. Oppeneer, T. P. dos Santos, A. Schleife, S. Meng, M. A. Sentef, M. Ruggenthaler, A. Rubio, I. Radu, M. Murnane et al., The 2021 ultrafast spectroscopic probes of condensed matter roadmap, J. Phys.: Condens. Matter 33, 353001 (2021).

[68] L. Trifunovic, O. Dial, M. Trif, J. R. Wootton, R. Abebe, A. Yacoby, and D. Loss, Long-Distance Spin-Spin Coupling via Floating Gates, Phys. Rev. X 2, 011006 (2012).

[69] G. Yang, C.-H. Hsu, P. Stano, J. Klinovaja, and D. Loss, Longdistance entanglement of spin qubits via quantum Hall edge states, Phys. Rev. B 93, 075301 (2016).

[70] J. Keeling, I. Klich, and L. S. Levitov, Minimal Excitation States of Electrons in One-Dimensional Wires, Phys. Rev. Lett. 97, 116403 (2006).

[71] J. Dubois, T. Jullien, F. Portier, P. Roche, A. Cavanna, Y. Jin, W. Wegscheider, P. Roulleau, and D. C. Glattli, Minimalexcitation states for electron quantum optics using levitons, Nature (London) 502, 659 (2013). 